\title{
At-home foot temperature monitoring reduced foot complications in high risk patients with diabetes
}

Lavery LA, Higgins KR, Lanctot DR, et al. Home monitoring of foot skin temperatures to prevent ulceration. Diabetes Care 2004;27:2642-7.

What is the effectiveness of at-home, infrared foot temperature monitoring for preventing foot complications in high risk patients with diabetes?

\section{METHODS}

Design: randomised controlled trial.

Allocation: $\{$ concealed\}.*

Blinding: $\{$ unblinded $\}{ }^{*}$

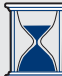

Follow up period: 6 months.

Setting: high risk diabetes foot clinics at the University of Texas Health Science Center, San Antonio, Texas.

Patients: 85 patients $18-80$ years of age (mean age $55 y, 51 \%$ men) who had diabetes (World Health Organization criteria) and were at high risk of developing diabetic foot ulcerations

(International Working Group on the Diabetic Foot risk group 2 or 3-ie, history of foot ulceration or lower extremity amputation, or peripheral sensory neuropathy with loss of protective sensation or foot deformity such as hallux valgus or claw toes). Exclusion criteria were open ulcers or open amputation sites, active Charco arthropathy, peripheral vascular disease, active foot infection, dementia, impaired cognitive function, or history of drug or alcohol use in the previous year.

$\mathbf{R}_{\mathbf{X}}$

Intervention: enhanced $(n=41)$ or standard therapy $(n=44)$. All patients received standard care, which included therapeutic

footwear, education, and foot evaluation by a podiatrist every 10-12 weeks. Patients in the enhanced therapy group also used a hand held, infrared skin thermometer (TempTouch, Xilas Medica, San Antonio, Texas) to measure the temperature of 6 predetermined sites on the sole of each foot (first, third, and fifth metatarsal head; great toe; central mid-foot; and heel) in the morning and evening; higher temperatures were considered to indicate ongoing inflammation. If the temperature of corresponding sites on the left and right foot differed by $>2.2^{\circ} \mathrm{C}$ $\left(4^{\circ} \mathrm{F}\right)$, patients were advised to contact a nurse case manager and to reduce the number of steps taken in the following days until the temperature difference was $<2.2^{\circ} \mathrm{C}$.

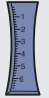

Outcomes: diabetic foot complications (incident foot ulcers, infections, Charcot fractures, and amputations).

Patient follow up: $100 \%$ (intention to treat analysis).

*Information provided by author.

\section{MAIN RESULTS}

Patients in the enhanced therapy group had fewer diabetic foot complications overall and fewer ulcers (table). The groups did not differ for Charcot fractures $(0 \vee 2)$, infections $(0 \vee 2$ [later required amputation]), or local foot amputations $(0 \vee 2)$.

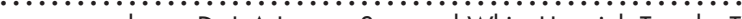
For correspondence: Dr L A Lavery, Scott and White Hosptial, Temple, TX, USA. Ilavery@swmail.sw.org

Source of funding: National Institutes of Health/National Institute of Diabetes and Digestive and Kidney Diseases.

\section{CONCLUSION}

In high risk patients with diabetes, at-home infrared, foot temperature monitoring reduced foot complications more than standard care.

A modified version of this abstract appears in Evidence-Based Medicine.

Enhanced therapy with at-home, infrared temperature monitoring $v$ standard therapy for preventing foot complications in high risk patients with diabetes without peripheral vascular disease*

\begin{tabular}{|c|c|c|c|c|}
\hline $\begin{array}{l}\text { Outcomes at } \\
6 \text { months }\end{array}$ & Enhanced & Standard & $\operatorname{RRR}(95 \% \mathrm{Cl})$ & NNT (CI) \\
\hline $\begin{array}{l}\text { Diabetic foot } \\
\text { complications } \\
\text { Ulcers }\end{array}$ & $\begin{array}{l}1 / 41 \\
(2.4 \%) \\
1 / 41 \\
(2.4 \%)\end{array}$ & $\begin{array}{l}9 / 44 \\
(20 \%) \\
7 / 44 \\
(16 \%)\end{array}$ & $\begin{array}{l}89 \% \text { (37 to } 98 \text { ) } \\
85 \% \text { (11 to } 97 \text { ) }\end{array}$ & $\begin{array}{l}6 \text { (3 to } 18) \\
8 \text { (4 to } 82 \text { ) }\end{array}$ \\
\hline
\end{tabular}

*Abbreviations defined in glossary; RRR, NNT, and Cl calculated from data in article.

\section{Commentary}

linicians use various measures to assess a patient's risk of developing a foot ulcer. Nevertheless, the increasing incidence of lower extremity amputations and the costs associated with both foot ulceration and amputation underscore the need for additional strategies for prevention and early detection.

Lavery et al provide a creative solution to this problem by having patients perform their own objective foot evaluations, beyond what normally would have been possible with visual inspection alone. The intervention not only appeared feasible, but was effective. Reporting of additional information such as the frequency of temperature abnormalities, patient compliance with reducing the number of steps taken, and the number of calls to the nurse and any subsequent clinical evaluation would provide further support for the effectiveness of the intervention. As the reported trial was a pilot study, more research is required.

Maintaining physical activity is essential in older individuals to prevent functional decline and in patients with diabetes to reduce cardiovascular risk. This further supports the need for patients and providers to carefully monitor for foot ulceration and modify activity as needed. As with any randomised trial, the intervention must be feasible for implementation in the larger population, which may have different levels of motivation. Importantly, the intervention did not adversely affect quality of life, making patient acceptability more likely. Additional measures of patient acceptability and feasibility in subsequent studies will assist in successfully translating these findings into clinical practice. Although additional monitoring behaviours may present challenges for patients and nurse educators, the magnitude of the problem demands that new and creative strategies be identified in order to reduce the risk of foot ulceration and lower extremity amputation.

Deborah Chyun, RN, PhD Yale University School of Nursing New Haven, Connecticut, USA 